Jürg A. Schifferli

\title{
Complement: a member of the innate immune system
}

Published online: 6 October 2005

(C) Springer-Verlag 2005

\section{Introduction}

During recent years, the field of innate immunity has exploded, particularly since the discovery of Toll-like receptors. It suddenly became evident to many immunologists that the initial inflammation induced by microorganisms was an essential trigger factor of the more elaborate specific immunity. The initial response is pattern recognition, whether on cell surfaces, as for Toll-like receptors, or in the fluid phase, as for complement and natural antibodies. Complement activation in the innate immune response is due to natural $\operatorname{IgM}$ antibodies and CRP, but is also directly due to mannan-binding lectin (protein) and deposition of $\mathrm{C} 3$ by the alternative pathway. Once acquired immunity has allowed the production of specific antibodies, complement again comes into play via the first recognized pathway of complement - the classical activation pathway-which occurs when C1q binds to the antigen-antibody complex. During ontogeny, the roles of protection against intruders and disposal of waste developed in parallel. In particular, apoptotic and necrotic cells have to be cleared. It is therefore not surprising that innate immunity, complement in particular, is also involved in helping to remove waste.

The knowledge gained in the field of complement has exploded in recent years, and this issue makes no claim to cover the topic completely. Rather, it is a selection of those fields in which there has been a development that might interest not only the immunologist but also the clinician who wants to understand more about specific aspects of diseases in which complement plays a central role.

The first complement deficiency to be identified was $\mathrm{C} 1$ inhibitor deficiency, although we now recognize that the clinical syndrome of angioedema is related to the inhibition of the kinin cascade by $\mathrm{C} 1$ inhibitor and not to its capacity to control the complement cascade. If we had to give a name to this molecule today, we might consider "kinin inhibitor" as more

J. A. Schifferli (四)

Department of Medicine, University Hospital Basel, Basel, Switzerland e-mail: j.schifferli@unibas.ch 
logical for clinicians, who first of all consider the pathophysiology of a disease. Cicardi reviews the field from basic knowledge about the immunopathological processes to the clinical aspects and indicates possible future treatment options. And the future appears to be near.

Anti-C1q autoantibodies were described initially by Agnello more than 30 years ago. Understanding of their role was hampered initially because $\mathrm{C} 1 \mathrm{q}$ binds to $\mathrm{IgG}$, and thus, the reverse reaction had to be indisputably established before the scientific world would accept the finding. Subsequently, the groups of Daha and Mannik established that such autoantibodies were frequently found in SLE, specifically in lupus nephritis, and were deposited in the kidney. This field is in development, and the finding that Clq deficiency (also knockout in mice) leads to an SLE-like disease underlines the major role of the C1q protein in SLE. Marten Trendelenburg takes us through the recent literature concerning antiC1q.

During the past decade, David Sacks has investigated the role of local synthesis of complement proteins in kidney diseases and, in particular, in renal transplantation. He has recently demonstrated the role of locally produced $\mathrm{C} 3$ in regulating alloreactivity and organ rejection. His article is a very thorough overview of the fascinating subject of renal production of complement.

The next article by Véronique Frémeaux-Bacchi deals with the hemolytic uremic syndrome (HUS) and complement abnormalities. The developments in this field in recent years have been fascinating, with the demonstration that specific mutations of factor $\mathrm{H}$ are associated with HUS, but also that MCP contributes to the protection against this devastating disease. The suggestion that factor I mutation can lead to HUS, as well as the finding in some rare cases that anti-factor $\mathrm{H}$ autoantibodies may be involved, provides additional support for the hypothesis that proper control of the alternative pathway is essential for protecting the renal glomerular endothelial cells against injury.

John Atkinson reviews the multiple aspects of "membrane cofactor protein" (MCP), which he calls CD46 in the title of his article. This is certainly due to the multiple aspects of MCP which are not directly related to its complement control activity, e.g., its interaction with many human pathogens or its role in inducing T-lymphocyte regulatory cells. This molecule first described by complementologists appears to have more facets than originally expected.

Following the description of mannan-binding lectin (MBP) many years ago, there has been much activity aimed at finding out how this protein activates the complement cascade. Many investigators successively described different "mannan-binding protein associated proteases" (MASPs), one of which-MASP2 - emerged as the most likely protease linking MBP to cleavage of C4 and C2. In this respect, the review of Rikke Sorensen and colleagues is timely, not only to describe the biochemistry of MASP2 but also for its possible role in preventing infection and autoimmunity, as suggested by the diseases associated with MASP2 deficiency in a unique patient.

Parasites appear to be capable, when it suits them, of stealing fragments of the human genome, for example, when the stolen fragment allows them to produce an inhibitor of complement activation. Indeed, the expression of a complement inhibitor may be protective for parasites, such as schistosomes, which live in the blood stream. Jameel Inal speculates on such a horizontal gene transfer for complement $\mathrm{C} 2$ receptor inhibitor trispanning (CRIT), a molecule he first found in schistosomes, then in trypanosomes, and finally in humans.

In the final article, David Pilzer and colleagues come back to a well-known phenomenon, i.e., the release of small vesicles by ectocytosis from cells which have been attacked by 
complement. These small vesicles remove the membrane attack complex from the cell surface, thereby protecting the cell. On the other hand, the biology of ectosomes has only recently attracted the interest of biologists. These small vesicles have functions such as release of mediators, transfer of information from one cell to another, and modulating inflammation and thrombosis.

It has been a privilege to bring together these different contributions, thanks to a wonderful group of investigators and friends. We thank them all and hope that the reviews provided will be helpful for clinicians and scientists alike. Finally, our thanks to Mrs. Jean Ringrose, who helped us to bring these reviews together in her very gentle way. 